\title{
New horizons in non-small-cell lung cancer patients with ipsilateral pleural dissemination (M1a): review of the literature
}

\author{
Hao Li", Taorui Liu", Zewen Sun, Zhenfan Wang, Xianping Liu, Fan Yang \\ Department of Thoracic Surgery, Centre of Thoracic Minimally Invasive Surgery, Peking University People’s Hospital, Beijing, China \\ Contributions: (I) Conception and design: H Li, F Yang; (II) Administrative support: F Yang; (III) Provision of study materials or patients: H Li, T \\ Liu; (IV) Collection and assembly of data: Z Wang, X Liu; (V) Data analysis and interpretation: Z Sun, T Liu; (VI) Manuscript writing: All authors. \\ (VII) Final approval of manuscript: All authors. \\ \#These authors contributed equally to this work. \\ Correspondence to: Fan Yang, MD. Department of Thoracic Surgery, Peking University People's Hospital, No. 11 Xizhimen South Street, Xicheng \\ District, Beijing 100044, China. Email: yangfan@pkuph.edu.cn.
}

\begin{abstract}
Non-small cell lung cancer (NSCLC) with ipsilateral pleural dissemination (pM1a) is generally contraindicated for surgery owing to the extremely poor survival. However, some studies have demonstrated that primary tumor resection (PTR) may prolong the survival of these patients. Besides, with the development of systemic therapy, it is still hard to decide the best therapy model for pM1a patients. Thus, we reviewed essential studies about NSCLC with pleural disease and summarized the progress of new techniques in recent years, trying to provide promising new horizons about the management of pM1a patients. Firstly, we suggest performing PTR for highly selected pM1a patients, combined with appropriate systemic therapies and follow-up strategies. Secondly, hyperthermic intrathoracic chemotherapy (HITHOC) can control the symptoms and prolong the survival of NSCLC patients with malignant pleural effusion (MPE). It could also combine with PTR together. Finally, application of genetic testing and circulating tumor DNA (ctDNA) monitoring may furthermore make it possible for personalized management of pM1a patients in the future.
\end{abstract}

Keywords: Ipsilateral pleural dissemination; primary tumor resection (PTR); hyperthermic intrathoracic chemotherapy (HITHOC); circulating tumor DNA monitoring (ctDNA monitoring)

Submitted Aug 29, 2020. Accepted for publication Jan 20, 2021.

doi: $10.21037 /$ atm-20-6188

View this article at: http://dx.doi.org/10.21037/atm-20-6188

\section{Introduction}

Lung cancer is the most commonly diagnosed cancer and the leading cause of cancer death around the world (1). Nonsmall-cell lung cancer (NSCLC) accounts for about $85 \%$ of lung cancer cases (2). Ipsilateral pleural dissemination is a special status of NSCLC which means tumor spread or metastasize to the ipsilateral pleural cavity, including malignant pleural/pericardial effusion and nodules, are defined as T4IIIB in the sixth edition of the Union for International Cancer Control (UICC) lung cancer staging system (3). However, further survival data showed those patients have a similar poor prognosis as contralateral lung nodules, with median survival time (MST) of 4-11.5 months and a 5-year survival rate of
$3-10 \%(4,5)$. Therefore, they are reclassified as M1a (stage IVA) together in the $7^{\text {th }}$ edition of the lung cancer staging system (4).

Owing to the poor survival, NSCLC patients with ipsilateral pleural dissemination lesions (pM1a) were recommended to receive systemic treatment according to all current guidelines, while surgical intervention has been considered as contraindicated (6-10). However, those patients have great heterogeneity regarding different pleural extension severity. Their clinical features range from a single nodule with localized pleural nodules to huge mass concomitant with diffused seeding or obvious malignant pleural effusion (MPE) (11). There is still no uniform 
therapy model applicable to all $\mathrm{pM} 1 \mathrm{a}$ patients, making the treatment of $\mathrm{pM} 1 \mathrm{a}$ patients full of controversy and challenges.

Recently, the survival of pM1a patients prolonged a lot with the development of systemic therapy, including targeted therapy and immune therapy. The progress of minimally invasive surgical technique makes surgery recapture an important role as a component of multimodal treatment. Besides, symptomatic treatments such as hyperthermic intrathoracic chemotherapy (HITHOC) achieve a positive effect and attract a lot of attention. Advances in diagnostic technology and genetic testing also lead researchers to explore genetic differences among pM1a patients (12), thereby making dynamic monitoring of tumor burden and personalized treatment possible in the future. In the following sections of this review, we will summarize the latest progress and provide promising new horizons about the management of $\mathrm{pM} 1 \mathrm{a}$ patients. We present the following article in accordance with the Narrative Review reporting checklist (available at http://dx.doi.org/10.21037/ atm-20-6188).

\section{Methods}

PubMed was searched for the resulting articles referenced to create this review. Additional articles were included to summarize the existing literature or provide sufficient background.

\section{Results}

\section{The value of primary tumor resection (PTR) of pM1a patients}

As early as the 1990s, Reyes et al. (13) firstly report the result of neoadjuvant chemotherapy followed by extrapleural pneumonectomy (EPP) to treat pleural dissemination disease. Yamaguchi et al. (14) then designed a phase II trial to assess the effect of preoperative concurrent chemoradiotherapy followed by EPP. However, because of the slow registration pace, this trial was prematurely terminated after involved only 11 patients. Long-term follow-up showed a 5-year disease-free survival (DFS) rate as $11.1 \%$ and 5 -year overall survival (OS) rate as $22.2 \%$. Recurrence developed in $88.9 \%$ of patients who underwent EPP. With the deep understanding of pleural dissemination, EPP had almost abandoned by surgeons. Thus in the last decade, surgeons concentrated more on cytoreductive surgery like PTR, but not radical resection for $\mathrm{pM} 1 \mathrm{a}$ patients, and combination of systemic therapy to prolong patients' OS.

Thoracic surgeons usually encountered two different clinical scenarios about pM1a NSCLC patients. The first one was patients who staged as resectable preoperatively were diagnosed pleural dissemination unexpectedly during surgery [clinical stage M0 (cM0)]. Surgeons should decide on whether to continue the operation or perform biopsy only. Generally, these patients were characteristic as localized pleural seeding or minor MPE couldn't be detected by preoperative imaging examination. So some surgeons tried to perform PTR for these patients instead of exploratory only. Another scenario was that MPE or pleural seeding had already been detected by preoperative staging procedures [clinical stage M1a (cM1a)]. In this scenario, surgical intervention was mainly performed for diagnostic biopsy and symptomatic relief. We will review the application of PTR in different types of clinical scenarios.

\section{For cMO patients}

The majority of studies on surgical intervention in pM1a patients have focused on $\mathrm{cM} 0$ patients. The earliest study on PTR for pM1a patients accidentally found during surgery was a multicenter retrospective study conducted by Ichinose et al. (15) in 2000. They involved a total of 227 cases of NSCLC patients found pleural dissemination at thoracotomy through a multicenter questionnaire survey of the Japan clinical oncology group (JCOG). One hundred and ninety-four patients of the whole cohort underwent surgical resection, while thoracotomy alone without resection was performed for the other 34 patients. The resection group put up a significantly better survival compared with exploratory group (5-year OS $14.9 \%$ vs. $0 \%$ ). The authors further analyzed the outcome of 100 patients with minimal carcinomatous pleuritis in PTR group. These patients yielded an unexpectedly good prognosis, with an MST of 20.6 months and the 3 - and 5-year survival rates of $31.8 \%$ and $22.8 \%$, respectively (16). This study proposed that PTR may bring survival benefits for patients with mild thoracic dissemination. However, Sawabata et al. (17) then reported a similar study but presented the opposite result. They found that patients with malignant minor pleural effusion detected at thoracotomy, even with complete gross resection of the tumor, had an MST of only 13 months. The opposite conclusions of the two similar studies might due to the differences in adjuvant treatment and surgical methods. 
Then, in the last two decades, several single-center retrospective studies with sample sizes ranging from 25 to 138 also reported outcomes of unexpected M1a patients undergoing PTR, with MSTs ranging from 15-64 months and 3 -year OSs ranging from $34.2 \%$ to $82.9 \%, 5$-year OSs ranging from $16.3-42.7 \%$, respectively (18-27). Most studies supported PTR as a significant prognostic factor for better survival for patients intraoperatively diagnosed as M1a. More details of these studies were listed in Table 1. There were trends in the increasing rate of minimally invasive surgery and normalized systemic treatment. For example, Li et al. (26) included 43 patients with lung adenocarcinoma with pleural seeding diagnosed unexpectedly during surgery using a prospectively collected database. PTR was performed in 30 patients while the remaining 13 patients underwent pleural nodule biopsy alone. The rate of videoassisted thoracoscopic surgery (VATS) was about $97.7 \%$ $(n=42)$. Twenty-nine patients received epidermal growth factor receptor tyrosine kinase inhibitor (EGFR-TKI) therapy (76\%). The patients undergoing PTR demonstrated an extremely good survival, with an MST of 64 months, 3 -year progression-free survival (PFS) of $44.5 \%$, and 3-year OS of $82.9 \%$. In the most recent study of Park et al. (27) from Seoul National University Hospital, 130 unexpected M1a patients were included. One hundred and ten patients (84.6\%) administered systemic treatment. The result showed that 5-year OS of the PTR group was superior to the biopsy group $(34.7 \%$ vs. $15.9 \%, \mathrm{P}=0.016)$. PTR only played a significant prognostic role in patients who received systemic treatment (5-year OS 40.0\% vs. 17.2\%, $\mathrm{P}=0.009$ ).

Besides these single-center retrospective studies, in 2016, Xu et al. (28) reported a meta-analysis reviewed nine retrospective studies with a total number of 861 unexpected pM1a patients to compare the survival benefit between exploration and PTR. The result showed PTR was a significant favorable predicted factor ( $\mathrm{HR}=0.443 ; 95 \% \mathrm{CI}$ : $0.344-0.571 ; \mathrm{P}<0.001)$. Female and lower $\mathrm{N}$ stage were also suggested to be beneficial prognostic factor. A most recent meta-analysis reported by Deng et al. (29) in 2020 included several newly published studies and drew a simple conclusion. These two meta-analyses were the strongest data supporting PTR superior to exploratory surgery for patients with unexpected pleural metastasis detected during operation till now.

\section{For cM1a patients}

Only a few studies concentrated on PTR for cM1a NSCLC patients. Several retrospective studies included M1a patients diagnosed both preoperatively and intraoperatively. Iida et al. (30) demonstrated that the 5 -year OS of 256 patients with pleural carcinomatosis who had received PTR was $33.1 \%$ in a multicenter questionnaire survey of JCOG. Best stage nodal status $(\mathrm{P}=0.002)$ and complete macroscopic resection $(\mathrm{P}=0.013)$ were independent predictors of survival. In 2015, Liu et al. (31) retrospectively analyzed the effectiveness of PTR in 80 M1a patients (including nine contralateral lung nodules) and found that the 5-year OS reached $31 \%$. These researches indicated that selected clinical M1a patients can still benefit from PTR combined with appropriate systemic treatment, suggesting that surgery can be used as a part of comprehensive multimodal treatment. Unfortunately, the sample sizes were limited, and no patients received genetic testing or targeted therapy.

The opening of public databases helped solve the limitation of sample size. In 2016, using the SEER database, Ren et al. (32) found that the prognosis of NSCLC patients with ipsilateral MPE after PTR may be better than expected (MST: 20 vs. 7 months, $\mathrm{P}<0.001$ ). Nevertheless, patients with pleural nodules and pericardial effusion were excluded from the study. Recently, we further analyzed 5,513 pM1a patients from the SEER database, among which 309 patients underwent PTR (33). Surgery was associated with improved OS in the entire cohort and surgery-recommended cohort, both before and after 1:3 propensity score matching (PSM). The multivariable analysis suggested that PTR was an independent favorable prognostic factor for both OS (HR: 0.56, 95\% CI: 0.48-0.54, $\mathrm{P}<0.001)$ and lung-cancer specific survival (SHR: 0.60, 95\% CI: 0.51-0.70, P<0.001). Further subgroup analysis showed that except for patients with pericardial effusion $(\mathrm{P}=0.065)$ or $\mathrm{N} 3$ disease $(\mathrm{P}=0.17)$, $\mathrm{PTR}$ was independently associated with prolonged survival in all subgroups (33). These studies are summarized in Table 2.

\section{Surgery for patients with different genetic mutations}

In the last 10 years, targeted therapy, such as TKIs for EGFR or anaplastic lymphoma kinase (ALK), significantly improved the survival of driver-oncogene positive stage IV NSCLC patients (35). According to previous literatures, adenocarcinoma is the most common histology subtype in $\mathrm{pM} 1 \mathrm{a}$ patients, with a prevalence of $64 \%$ to $90.9 \%$ $(15,17-25,27,30,31,33,34)$. Thus, pM1a patients are likely to benefit from targeted therapy. Li et al. (26) reported an extremely good survival of lung adenocarcinoma patients with unexpected pleural seeding undergoing PTR (MST: 


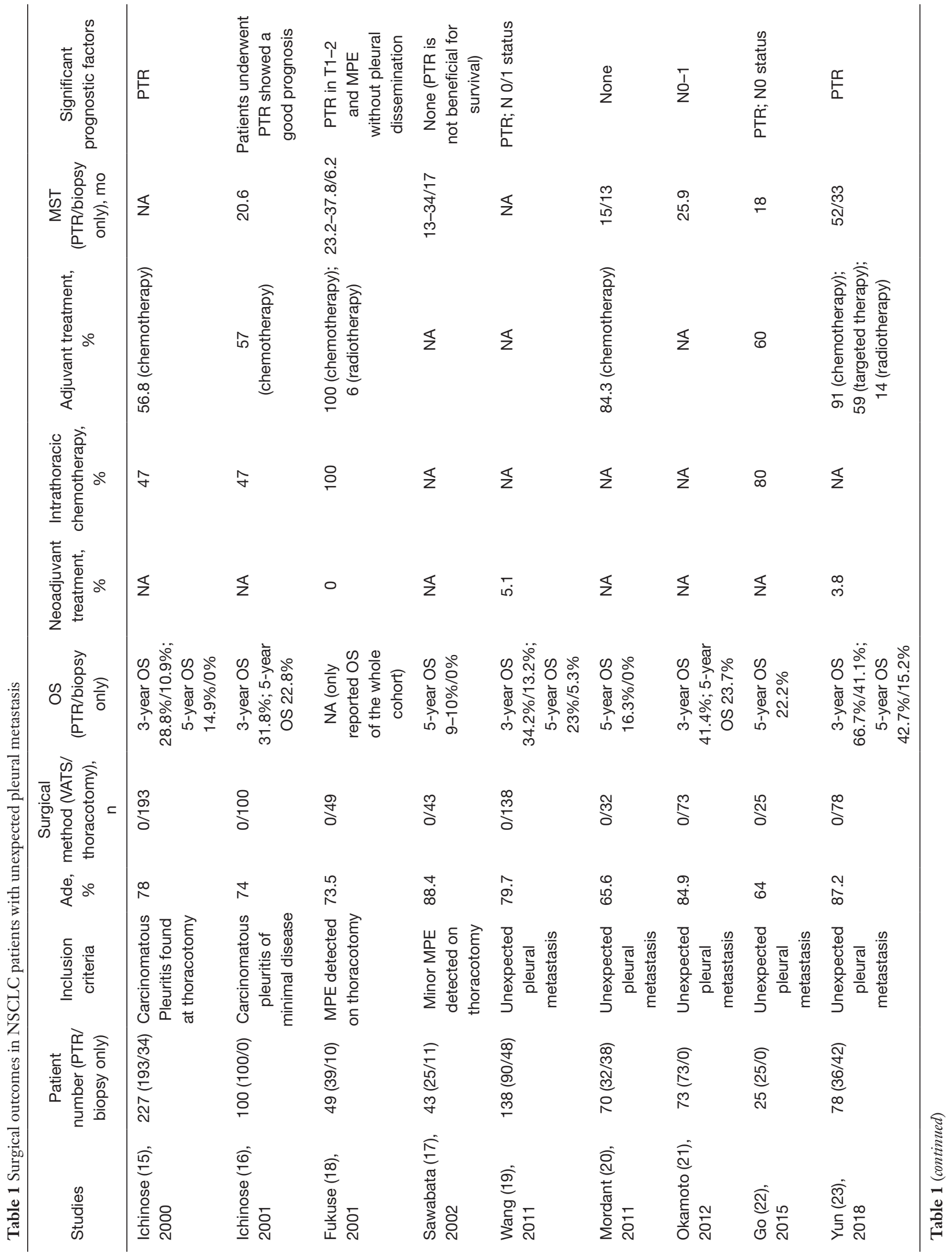




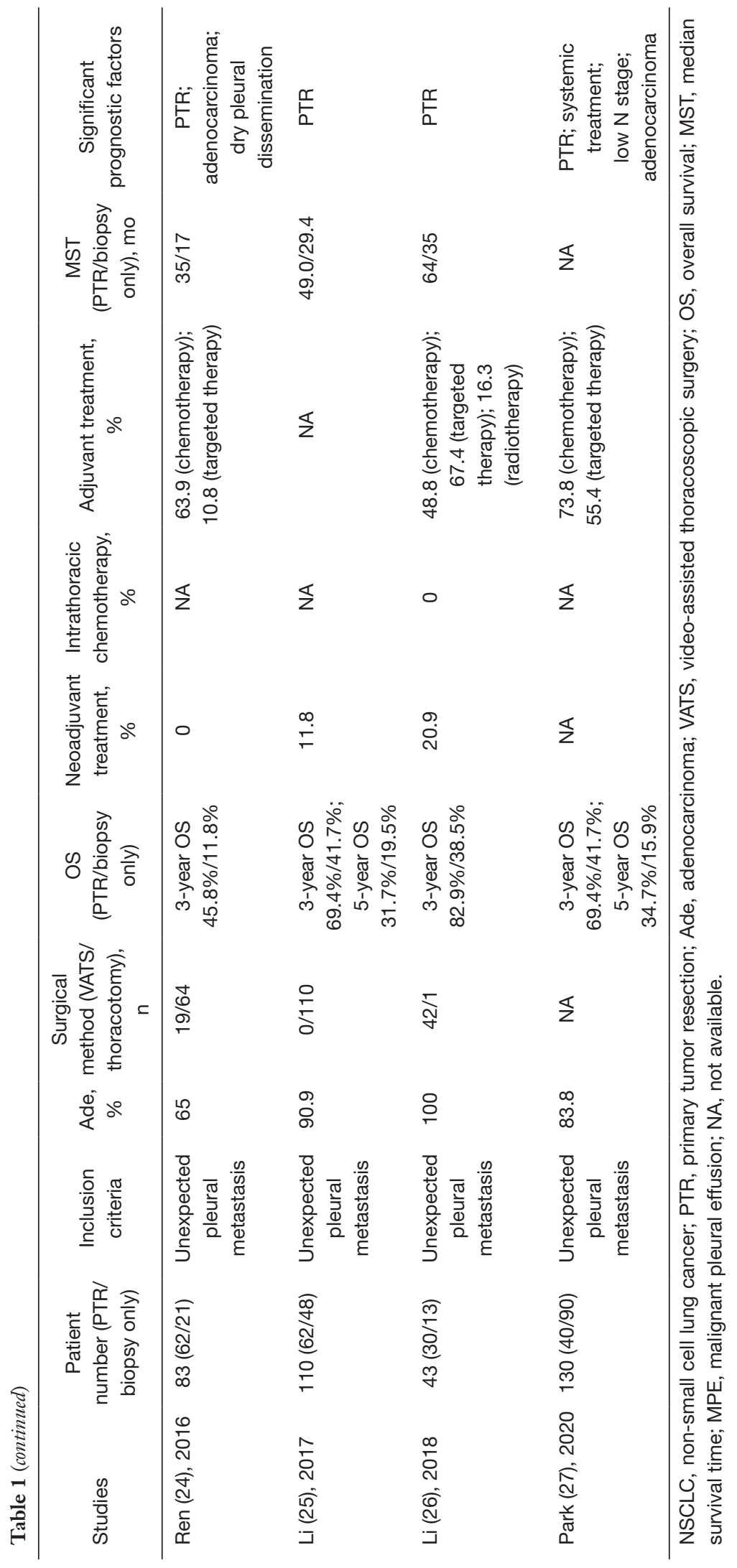


Table 2 Surgical outcomes in pM1a patients (without dividing preoperative or intraoperative M1a)

\begin{tabular}{|c|c|c|c|c|c|c|c|c|c|c|}
\hline Studies & $\begin{array}{c}\text { Patient } \\
\text { number } \\
\text { (PTR/biopsy } \\
\text { only), n }\end{array}$ & $\mathrm{cM0} / \mathrm{cM} 1$ & $\begin{array}{l}\text { Inclusion } \\
\text { criteria }\end{array}$ & $\begin{array}{c}\text { Ade, } \\
\%\end{array}$ & $\begin{array}{c}\text { EGFR-TKIs, } \\
\qquad \%\end{array}$ & $\begin{array}{c}\text { OS } \\
\text { (PTR/biopsy } \\
\text { only) }\end{array}$ & $\begin{array}{l}\text { Neoadjuvant } \\
\text { treatment, \% }\end{array}$ & $\begin{array}{c}\text { Adjuvant } \\
\text { treatment, \% }\end{array}$ & $\begin{array}{c}\text { MST } \\
\text { (PTR/biopsy } \\
\text { only), mo }\end{array}$ & $\begin{array}{c}\text { Significant } \\
\text { prognostic } \\
\text { factors }\end{array}$ \\
\hline $\begin{array}{l}\text { Hanagiri } \\
(34), 2012^{\dagger}\end{array}$ & $17(17 / 0)$ & $13 / 4$ & $\begin{array}{l}\text { Stage IV } \\
\text { NSCLC }\end{array}$ & NA & NA & $\begin{array}{c}\text { 5-year OS } \\
25.3 \%\end{array}$ & NA & NA & NA & NA \\
\hline $\begin{array}{l}\text { Ren (32), } \\
2016\end{array}$ & $\begin{array}{c}2,217 \\
(128 / 2,089)\end{array}$ & NA & MPE & 50 & NA & NA & NA & NA & $20 / 7$ & PTR \\
\hline $\begin{array}{l}\mathrm{Li}(33), \\
2019\end{array}$ & $\begin{array}{c}5,513 \\
(309 / 5,204)\end{array}$ & NA & pM1a & 71.6 & NA & $\begin{array}{c}5 \text {-year OS } \\
9-10 \% / 0 \%\end{array}$ & NA & NA & $20 / 8$ & PTR \\
\hline
\end{tabular}

${ }^{\dagger}$, This study included 36 patients undergoing surgical resection for stage IV NSCLC, of which 17 patients compared with pleural dissemination. So some data was not available for individual M1a patients. ${ }^{\ddagger}$, this study included also included nine patients with contralateral lung nodules. PTR, primary tumor resection; Ade, adenocarcinoma; EGFR, epidermal growth factor receptor; TKI, tyrosine kinase inhibitor; OS, overall survival; MST, median survival time; NSCLC, non-small cell lung cancer; ECOG, Eastern Cooperative Oncology Group; NA, not available; MPE, malignant pleural effusion.

64 months). One important reason for the inspiring result was their good use of EGFR-TKIs (76\%). However, do we still need to perform PTR among EGFR mutation-positive patients who can have excellent survival with only EGFRTKIs therapy? Since previous studies had demonstrated that tumor heterogeneity played an important role in acquired resistance of EGFR/ALK-TKIs (36), could PTR reduce tumor burden and heterogeneity to enhance the effect of targeted therapy? Whether PTR is beneficial for different driver oncogene-status patients is an interesting unanswered question.

Our recent study tried to answer it. We included 105 pM1a patients treated in our department between 2006 and 2016, of which 70 patients received genetic testing. Fiftyfive patients were positive for driver-oncogene mutations, while the other 50 patients with oncogenes unknown/ negative. A total of 54 patients received targeted therapy (except for one patient with ROS1 arrangement refused targeted therapy). In the targeted therapy subgroup, PTR did not prolong OS (MST: 57.1 vs. 50.4 months, $\mathrm{P}=0.840$ ).
However, in the non-targeted therapy group, PTR significantly prolonged survival (MST: $39.8 v s .14 .2$ months, $\mathrm{P}=0.002$ ) (37). This single-center retrospective study suggested PTR conferred a better outcome in M1a patients who were not candidates for targeted therapy. However, the evidence is still insufficient, and further researches are needed to solve this question. It may also be one of the future research directions in this field.

At present, there are several problems in the researches of PTR for pM1a patients: (I) most studies were retrospective studies with limited sample size, providing not strong enough evidence to change the routine clinical practice; (II) the criteria of appropriate pM1a patients for PTR are still unclear. Although previous studies provided some favorable prognostic factors for $\mathrm{pM} 1 \mathrm{a}$ patients performed PTR, it is still difficult to generalize them to all pM1a patients for the circumscribed conclusions; (III) there are few studies regard to the most appropriate surgical resection extension under the multimodal treatment model. Surgeons are still confused about 
whether to perform lobectomy or sublobar resection with unexpected pleural metastasis detected during operation. Future larger sample prospective clinical trials are still needed to solve the above questions.

\section{HITHOC for M1a NSCLC patients}

Traditional symptomatic treatment for pleural dissemination includes thoracentesis, thoracic drainage, pleural fixation and so on. Effective control of pleural effusion in MPE patients treated by these therapies was about $82-100 \%$ according to the previous literature $(38,39)$. Mainly disadvantages of these treatments were high rate of effusion recurrence and complications. Thoracic hyperthermic perfusion therapy was an emerging technology that used physical methods to heat the perfusion fluid and pour it into the thoracic cavity. This technique could keep the thoracic cavity at a certain temperature and persist for a while, achieving the effect of killing tumor cells and preventing MPE (40). Adding platinum or other chemotherapeutics to the hyperthermic perfusion can enhance the therapeutic effect. This was called HITHOC. The mechanism of HITHOC consist reducing the activity of malignant cells by hyperphysiological temperature and exposing tumor cell to high concentrations of chemotherapeutics (41).

Treating NSCLC patients with MPE were one of the important applications of HITHOC. Several studies reported the result of HITHOC using in M1a patients. In 2017, Hu et al. (42) reported a retrospective study involved 54 cases of pM1a patients with MPE. HITHOC was performed under VATS, and pleural biopsies were performed both before and after treatment. All patients received complete response of pleural effusion. Apoptosis of malignant tissue can be microscopically detected after HITHOC. Further follow-up showed good prognosis, with an MST of 21.7 months and 1 -year survival rate of $74.1 \%$. Zhou et al. (43) conducted a systematic review and meta-analysis comparing the survival of MPE patients between HITHOC group and non-HITHOC group. The results showed that HITHOC significantly prolonged survival of patients with MPE (Hedges $g=0.763$, $\mathrm{P}<0.001)$. However, the specific rate of NSCLC was unclear. A most recent prospective randomized trial reported by Kleontas et al. (44) evaluated the survival difference between HITHOC and talc pleurodesis for NSCLC patients with ipsilateral MPE. They included 20 patients for each group, and no significant difference was found between two groups (MST: 8 vs. 9 months, $\mathrm{P}=0.843$ ). This study suggested both HITHOC and talc pleurodesis were equally effective and safe options. The above studies all supported that HITHOC as an effective treatment for pM1a patients with MPE.

HITHOC under VATS can combine cytoreduction, hyperthermia, and chemotherapy, achieving the purpose of diagnosis and treatment at the same time. Thoracic surgeons sometimes see a direct invasion of the parietal pleura but sometimes they see intraoperatively disseminated nodules on the parietal and mediastinal pleura which can vary from 3,4 to more than 20 . When there is a direct invasion and a few disseminated nodules, the decision to proceed with surgery is easy; on the opposite, it is more difficult when there are many disseminated nodules on parietal and mediastinal pleura, or with subsequent MPE. In this circumstances, we prefer to go ahead with the operation if the patient is suitable for HITHOC procedure.

Therefore, some thoracic surgeons tried to compare whether PTR under VATS plus HITHOC can bring more survival benefits to pM1a patients. Yi et al. (45) reported a retrospective study that involved $33 \mathrm{pM}$ a patients (22 cases of $\mathrm{cM} 1 \mathrm{a}$ and 11 cases of $\mathrm{cM} 0$ ). Twenty-three cases of the whole cohort underwent HITHOC after PTR, while the other ten patients only underwent PTR. The results showed that the OS of the HITHOC group was significantly longer than that of the PTR alone group (5-year OS $38.6 \%$ vs. $37.5 \%, \mathrm{P}=0.045$ ). In 2019, a systematic review reported by Migliore $e t$ al. (46) shown favorable outcomes for N0-1 NSCLC patients with MPE undergoing PTR plus HITHOC, with an MST of 18 months and 2-year OS of $28.5 \%$. These studies suggested that PTR combined with HITHOC can be an effective surgical intervention for selected $\mathrm{pM} 1 \mathrm{a}$ patients to improve the quality of life and prolong life expectancy. Studies about HITHOC for pM1a patients were listed in Table 3.

Taken together of those previous studies, we would like to propose a surgical therapeutic strategy for M1a NSCLC patients as follows. (I) For cM0 but pathological staging pM1a patients, which means "unexpected" pleural dissemination during surgery, surgeons should try to resect the main tumor to prolong patients' survival and harvest enough tissue for gene testing. (II) For clinical staging pM1a patients, such as MPE confirmed by cytopathology, surgical intervention should be carefully included as an important option of multimodal therapy regimens, especially for patients with negative driver mutations and lymph node-negative (37). (III) The surgery would only be considered in operable patients, and should be relatively contraindicated for patients with N2/N3 metastasis or malignant pericardial effusion because of the poor survival after PTR (33). (IV) To 
Table 3 Effect of HITHOC for pM1a patients

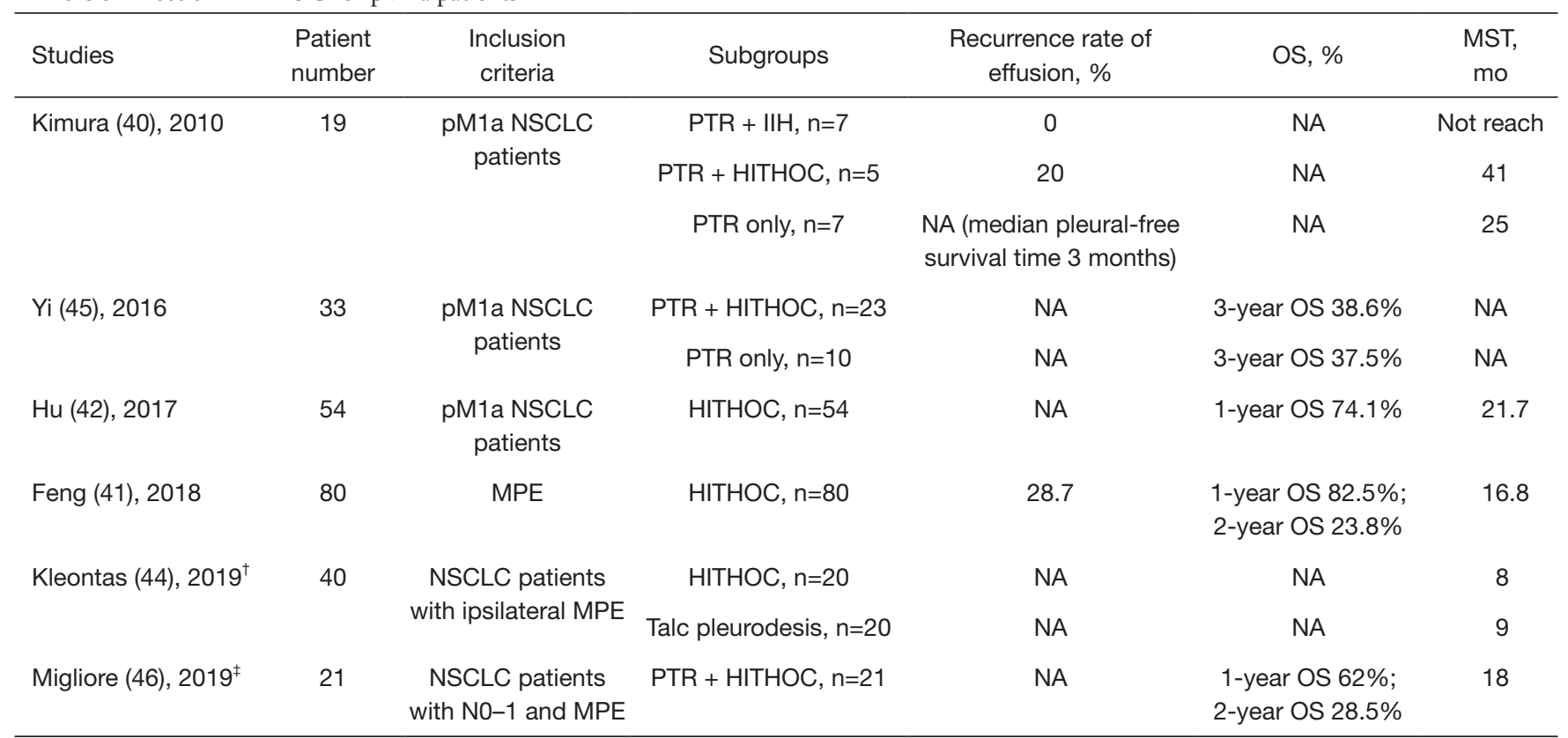

${ }^{\dagger}$, this study contained 65 NSCLC patients; ${ }^{\ddagger}$, this study was a systematic review. HITHOC, hyperthermic intrathoracic chemotherapy; IIH, intraoperative intrathoracic hyperthermotherapy; OS, overall survival; MST, median survival time; MPE, malignant pleural effusion; PTR, primary tumor resection; NA, not available.

make patients receive systemic therapy sooner, thoracic surgeons should consider the VATS technique superior to thoracotomy. Sublobar resection or lobectomy may be superior to pneumonectomy with a similar survival benefit but fewer complications (33). (V) Apart from the resection of primary tumor, intrathoracic hyperthermotherapy or hyperthermo-chemotherapy should also be considered to prevent MPE and improve the quality of life of pM1a patients with malignant pleural dissemination (46).

\section{Management of post-surgery pM1a NSCLC patients}

Although most studies recommended systemic treatment after PTR, such as chemotherapy, targeted therapy, and immune therapy, some investigators proposed a wait-and-see treatment strategy due to the observation of gradual progression in some pM1a patients (12). Chen et al. (12) retrospectively studied 131 pM1a patients with a diagnosis of special pleural dissemination lesions after PTR. The patients who received chemotherapy or targeted therapy after the wait-and-see strategy showed better OS than for those who received systematic therapy immediately (not reached vs. 41.7 months, HR: 0.45, 95\% CI: $0.23-0.88, \mathrm{P}=0.019)$. The whole-exome sequencing analysis of the ten patients with dramatic progression and 13 patients with gradual progression showed that low genomic instability index was significantly associated with better PFS $(\mathrm{P}=0.016)(12)$. This study demonstrated that significant heterogeneity of disease progression exists within $\mathrm{pM} 1 \mathrm{a}$ patients. The wait-andsee strategy could be considered for special pM1a patients with gradual progression.

The critical issues of the appropriate treatment strategy selection for post-surgery pM1a patients are finding out patients with rapidly progressive diseases. Particularly, the traditional radiological examinations, such as chest CT and PET-CT, could not evaluate tumor burden well due to the loss of evaluable lesions after main tumor resection. Recently, circulating tumor DNA (ctDNA), namely short DNA fragments shed by tumor cells from multiple tumor regions, has been proven capable of accurately assessing minimal residual disease (MRD) and identifying patients who might be at higher risk of relapse (47). Recently, we prospectively studied perioperative dynamic changes of ctDNA (DYNAMIC) and revealed that the median ctDNA half-life was only 35.0 minutes $(48,49)$. The recurrence-free survival of patients with detectable and undetectable ctDNA concentrations on the third day after R0 resection was 278 and 637 days, respectively ( $\mathrm{P}=0.002)$ (49). Longitudinal 


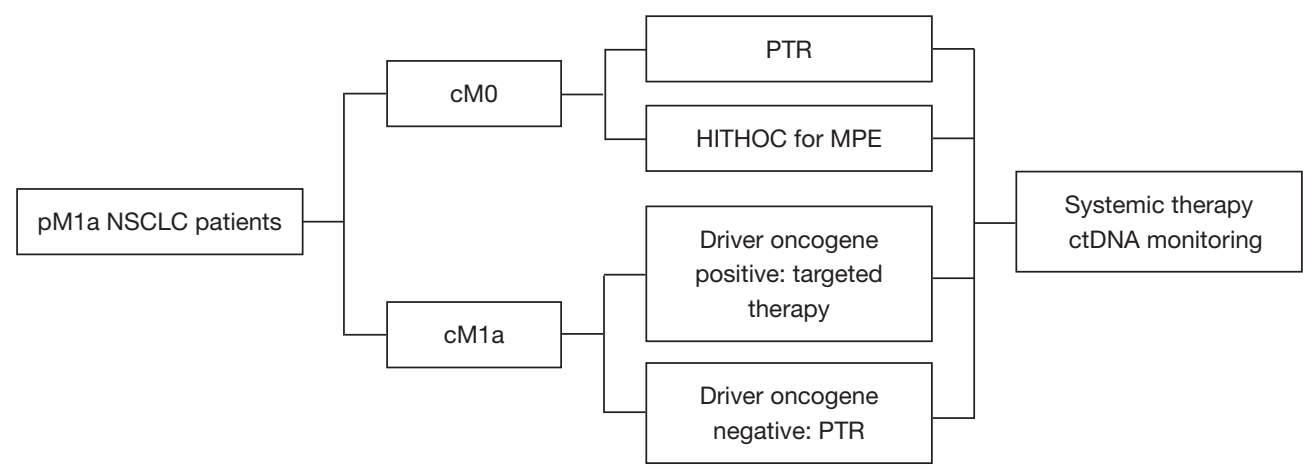

Figure 1 Management of pM1a patients. NSCLC, non-small cell lung cancer; cM0, clinical stage M0; cM1a, clinical stage M1a; PTR, primary tumor resection; HITHOC, hyperthermic intrathoracic chemotherapy; MPE, malignant pleural effusion; ctDNA, circulating tumor DNA.

ctDNA monitoring showed that the ctDNA changes were correlated with treatment response well and predicted disease progression accurately (49). ctDNA monitoring could be applied to guide the appropriate treatment strategy selection for pM1a patients in the future, especially for patients received main tumor resection. Hence, we conducted an open-label, multicenter, prospective, observational study in which advanced NSCLC patients will be recruited and longitudinal changes in ctDNA levels with changes in radiology will be assessed in order to determine the clinical utility of ctDNA monitoring (50). The study is still in progress, and we hope its result can provide more guidance for treatment strategies development and dynamic monitoring of pM1a patients. Surgical therapeutic strategy and post-surgery management of pM1a patients are summarized as Figure 1.

\section{Conclusions}

Overall, NSCLC patients with ipsilateral pleural dissemination have a poor prognosis, and there is still no uniform therapy model for these patients. By reviewing the previous studies, we proposed the treatment of $\mathrm{pM} 1 \mathrm{a}$ patients in different clinical scenarios. We suggested that surgery could be used in multimodal therapy for carefully selected pM1a patients. HITHOC can work as an effective symptomatic treatment for MPE caused by NSCLC. The development of genetic testing and ctDNA monitoring might make personalized treatment and follow-up possible in the future. However, previous studies on M1a are mostly retrospective, with certain bias and limited sample sizes. Such concerns should be considered in future multi- institutional randomized controlled studies.

\section{Acknowledgments}

We would like to thank all the mumbers of our department for their kind support.

Funding: None.

\section{Footnote}

Provenance and Peer Review: This article was commissioned by the Guest Editor (Marcello Migliore) for the series "Hyperthermic Intraoperative Chemotherapy (HITHOC) in thoracic surgical oncology" published in Annals of Translational Medicine. The article has undergone external peer review.

Reporting Checklist: The authors have completed the Narrative Review reporting checklist. Available at http:// dx.doi.org/10.21037/atm-20-6188

Conflicts of Interest: All authors have completed the ICMJE uniform disclosure form (available at http://dx.doi. org/10.21037/atm-20-6188). The series "Hyperthermic Intraoperative Chemotherapy (HITHOC) in thoracic surgical oncology" was commissioned by the editorial office without any funding or sponsorship. The authors have no other conflicts of interest to declare.

Ethical Statement: The authors are accountable for all aspects of the work in ensuring that questions related to the accuracy or integrity of any part of the work are 
appropriately investigated and resolved.

Open Access Statement: This is an Open Access article distributed in accordance with the Creative Commons Attribution-NonCommercial-NoDerivs 4.0 International License (CC BY-NC-ND 4.0), which permits the noncommercial replication and distribution of the article with the strict proviso that no changes or edits are made and the original work is properly cited (including links to both the formal publication through the relevant DOI and the license). See: https://creativecommons.org/licenses/by-nc-nd/4.0/.

\section{References}

1. Bray F, Ferlay J, Soerjomataram I, et al. Global cancer statistics 2018: GLOBOCAN estimates of incidence and mortality worldwide for 36 cancers in 185 countries. CA Cancer J Clin 2018;68:394-424.

2. Pilleron S, Sarfati D, Janssen-Heijnen M, et al. Global cancer incidence in older adults, 2012 and 2035: a population-based study. Int J Cancer 2019;144:49-58.

3. Mountain CF. Revisions in the international system for staging lung cancer. Chest 1997;111:1710-7.

4. Postmus PE, Brambilla E, Chansky K, et al. The IASLC Lung Cancer Staging Project: proposals for revision of the $M$ descriptors in the forthcoming (seventh) edition of the TNM classification of lung cancer. J Thorac Oncol 2007;2:686-93.

5. Goldstraw P, Chansky K, Crowley J, et al. The IASLC lung cancer staging project: proposals for revision of the TNM stage groupings in the forthcoming (eighth) edition of the TNM classification for lung cancer. J Thorac Oncol 2016;11:39-51.

6. David Sea. National comprehensive cancer network clinical practice guidelines in oncology: non-small cell lung cancer version 2.2020 Available online: http://www.nccn. org/professionals/physician_gls/pdf/nscl.Pdf. Accessed Feburary 1, 2020.

7. Ettinger DS, Aisner DL, Wood DE, et al. NCCN guidelines insights: non-small cell lung cancer, version 5.2018. J Natl Compr Canc Netw 2018;16:807-21.

8. Planchard D, Popat S, Kerr K, et al. Metastatic non-small cell lung cancer: ESMO Clinical Practice Guidelines for diagnosis, treatment and follow-up. Ann Oncol 2018;29:iv192-237.

9. Hanna N, Johnson D, Temin S, et al. Systemic therapy for stage IV non-small-cell lung cancer: american society of clinical oncology clinical practice guideline update summary. J Oncol Pract 2017;13:832-7.

10. Akamatsu H, Ninomiya K, Kenmotsu H, et al. The Japanese Lung Cancer Society Guideline for non-small cell lung cancer, stage IV. Int J Clin Oncol 2019;24:731-70.

11. Heffner JE, Klein JS. Recent advances in the diagnosis and management of malignant pleural effusions. Mayo Clin Proc 2008;83:235-50.

12. Chen $Y$, Tang WF, Lin H, et al. Wait-and-see treatment strategy could be considered for lung adenocarcinoma with special pleural dissemination lesions, and low genomic instability correlates with better survival. Ann Surg Oncol 2020;27:3808-18.

13. Reyes L, Parvez Z, Regal AM, et al. Neoadjuvant chemotherapy and operations in the treatment of lung cancer with pleural effusion. J Thorac Cardiovasc Surg 1991;101:946-7.

14. Yamaguchi M, Ichinose $Y$, Shimamatsu S, et al. Preoperative concurrent chemoradiotherapy followed by extrapleural pneumonectomy for patients with nonsmall cell lung cancer with malignant pleural effusion and/or pleural nodules: Ten-year results of a prematurely terminated single institute phase II trial. Surg Oncol 2015;24:78-83.

15. Ichinose $Y$, Tsuchiya R, Koike $T$, et al. The prognosis of patients with non-small cell lung cancer found to have carcinomatous pleuritis at thoracotomy. Surg Today 2000;30:1062-6.

16. Ichinose $\mathrm{Y}$, Tsuchiya R, Koike T, et al. Prognosis of resected non-small cell lung cancer patients with carcinomatous pleuritis of minimal disease. Lung Cancer 2001;32:55-60.

17. Sawabata N, Matsumura A, Motohiro A, et al. Malignant minor pleural effusion detected on thoracotomy for patients with non-small cell lung cancer: is tumor resection beneficial for prognosis? Ann Thorac Surg 2002;73:412-5.

18. Fukuse T, Hirata T, Tanaka F, et al. The prognostic significance of malignant pleural effusion at the time of thoracotomy in patients with non-small cell lung cancer. Lung Cancer 2001;34:75-81.

19. Wang BY, Wu YC, Hung JJ, et al. Prognosis of nonsmall-cell lung cancer with unexpected pleural spread at thoracotomy. J Surg Res 2011;169:e1-5.

20. Mordant P, Arame A, Foucault C, et al. Surgery for metastatic pleural extension of non-small-cell lung cancer. Eur J Cardiothorac Surg 2011;40:1444-9.

21. Okamoto T, Iwata T, Mizobuchi T, et al. Pulmonary resection for lung cancer with malignant pleural disease first detected at thoracotomy. Eur J Cardiothorac Surg 
2012;41:25-30.

22. Go T, Misaki N, Matsuura N, et al. Role of surgery in multi-modality treatment for carcinomatous pleuritis in patients with non-small cell lung cancer. Surg Today 2015;45:197-202.

23. Yun JK, Kim MA, Choi CM, et al. Surgical outcomes after pulmonary resection for non-small cell lung cancer with localized pleural seeding first detected during surgery. Thorac Cardiovasc Surg 2018;66:142-9.

24. Ren YJ, She YL, Dai CY, et al. Primary tumour resection showed survival benefits for non-small-cell lung cancers with unexpected malignant pleural dissemination. Interact Cardiovasc Thorac Surg 2016;22:321-6.

25. Li S, Zhang S, Huang M, et al. Management of occult malignant pleural disease firstly detected at thoracotomy for non-small cell lung cancer patients. J Thorac Dis 2017;9:3851-8.

26. Li C, Kuo SW, Hsu HH, et al. Lung adenocarcinoma with intraoperatively diagnosed pleural seeding: Is main tumor resection beneficial for prognosis? J Thorac Cardiovasc Surg 2018;155:1238-49.e1.

27. Park S, Chung Y, Lee HJ, et al. The role of primary tumor resection in patients with pleural metastasis encountered at the time of surgery. Korean J Thorac Cardiovasc Surg 2020;53:114-20.

28. Xu Y, Chen N, Wang Z, et al. Should primary tumor be resected for non-small cell lung cancer with malignant pleural disease unexpectedly found during operation?-a systemic review and meta-analysis. J Thorac Dis 2016;8:2843-52.

29. Deng HY, Zheng X, Zhu DX, et al. Is surgical resection of primary tumour superior to exploratory thoracotomy without resection in treating lung cancer patients with unexpected pleural metastasis detected during operation? Interact Cardiovasc Thorac Surg 2020;30:582-7.

30. Iida T, Shiba M, Yoshino I, et al. Surgical intervention for non-small-cell lung cancer patients with pleural carcinomatosis: results from the Japanese Lung Cancer Registry in 2004. J Thorac Oncol 2015;10:1076-82.

31. Liu T, Liu H, Wang G, et al. Survival of M1a non-small cell lung cancer treated surgically: a retrospective singlecenter study. Thorac Cardiovasc Surg 2015;63:577-82.

32. Ren Y, Dai C, Shen J, et al. The prognosis after contraindicated surgery of NSCLC patients with malignant pleural effusion (M1a) may be better than expected. Oncotarget 2016;7:26856-65.

33. Li H, Sun Z, Yang F, et al. Primary tumour resection in non-small-cell lung cancer patients with ipsilateral pleural dissemination (M1a): a population-based study. Eur J Cardiothorac Surg 2019;55:1121-9.

34. Hanagiri T, Takenaka M, Oka S, et al. Results of a surgical resection for patients with stage IV non-small-cell lung cancer. Clin Lung Cancer 2012;13:220-4.

35. Kris MG, Johnson BE, Berry LD, et al. Using multiplexed assays of oncogenic drivers in lung cancers to select targeted drugs. JAMA 2014;311:1998-2006.

36. Suda K, Murakami I, Sakai K, et al. Heterogeneity in resistance mechanisms causes shorter duration of epidermal growth factor receptor kinase inhibitor treatment in lung cancer. Lung Cancer 2016;91:36-40.

37. Li H, Liu T, Sun Z, et al. Primary tumor resection of non-small cell lung cancer patients with ipsilateral pleural dissemination (M1a) in the era of targeted therapy. Thoracic Cancer, 2020;11:3213-22.

38. Roberts ME, Neville E, Berrisford RG, et al. Management of a malignant pleural effusion: British Thoracic Society Pleural Disease Guideline 2010. Thorax 2010;65 Suppl 2:ii32-40.

39. Tremblay A, Michaud G. Single-center experience with 250 tunnelled pleural catheter insertions for malignant pleural effusion. Chest 2006;129:362-8.

40. Kimura M, Tojo T, Naito H, et al. Effects of a simple intraoperative intrathoracic hyperthermotherapy for lung cancer with malignant pleural effusion or dissemination. Interact Cardiovasc Thorac Surg 2010;10:568-71.

41. Feng X, Zhu L, Xiong X, et al. Therapeutical effect of intrapleural perfusion with hyperthermic chemotherapy on malignant pleural effusion under video-assisted thoracoscopic surgery. Int J Hyperthermia 2018;34:479-85.

42. Hu R, Jiang H, Li H, et al. Intrapleural perfusion thermo-chemotherapy for pleural effusion caused by lung carcinoma under VATS. J Thorac Dis 2017;9:1317-21.

43. Zhou H, Wu W, Tang X, et al. Effect of hyperthermic intrathoracic chemotherapy (HITHOC) on the malignant pleural effusion: a systematic review and meta-analysis. Medicine (Baltimore) 2017;96:e5532.

44. Kleontas A, Sioga A, Pandria N, et al. Clinical factors affecting the survival of patients diagnosed with non-small cell lung cancer and metastatic malignant pleural effusion, treated with hyperthermic intrathoracic chemotherapy or chemical talc pleurodesis: a monocentric, prospective, randomized trial. J Thorac Dis 2019;11:1788-98.

45. Yi E, Kim D, Cho S, et al. Clinical outcomes of cytoreductive surgery combined with intrapleural perfusion of hyperthermic chemotherapy in advanced lung 
adenocarcinoma with pleural dissemination. J Thorac Dis 2016;8:1550-60.

46. Migliore M, Nardini M. Does cytoreduction surgery and hyperthermic intrathoracic chemotherapy prolong survival in patients with N0-N1 nonsmall cell lung cancer and malignant pleural effusion? Eur Respir Rev 2019;28:190018.

47. Abbosh C, Birkbak NJ, Wilson GA, et al. Phylogenetic ctDNA analysis depicts early-stage lung cancer evolution. Nature 2017;545:446-51.

48. Chen K, Zhao H, Yang F, et al. Dynamic changes of

Cite this article as: Li H, Liu T, Sun Z, Wang Z, Liu X, Yang F. New horizons in non-small-cell lung cancer patients with ipsilateral pleural dissemination (M1a): review of the literature. Ann Transl Med 2021;9(11):959. doi: 10.21037/atm-20-6188 circulating tumour DNA in surgical lung cancer patients: protocol for a prospective observational study. BMJ Open 2018;8:e019012.

49. Chen K, Zhao H, Shi Y, et al. Perioperative dynamic changes in circulating tumor DNA in patients with lung cancer (DYNAMIC). Clin Cancer Res 2019;25:7058-67.

50. Gao Z, Shang Y, Wang X, et al. Application of circulating tumor DNA for dynamic monitoring of advanced nonsmall cell lung cancer treatment response: an open-label, multicenter, prospective, observational study protocol. Thorac Cancer 2019;10:1310-5. 